\title{
To drop or not to drop the antiplatelet agent, that is the question for patients with atrial fibrillation and chronic coronary syndrome undergoing percutaneous coronary intervention
}

\author{
Yongcheol Kim ${ }^{1}$, Thomas W. Johnson ${ }^{2}$, Young-Hoon Jeong ${ }^{3}$ \\ ${ }^{1}$ Division of Cardiology, Chonnam National University Hospital, Gwangju, Republic of Korea \\ ${ }^{2}$ Department of Cardiology, Bristol Heart Institute, Bristol, United Kingdom \\ ${ }^{3}$ Department of Internal Medicine, Gyeongsang National University School of Medicine and Cardiovascular \\ Center, Gyeongsang National University Changwon Hospital, Changwon, Republic of Korea
}

\section{Article p. 8}

The prevalence of atrial fibrillation $(\mathrm{AF})$ in patients who undergo percutaneous coronary intervention $(\mathrm{PCI})$ is $5-15 \%$, and optimal antithrombotic therapy should be decided based on a balance be-

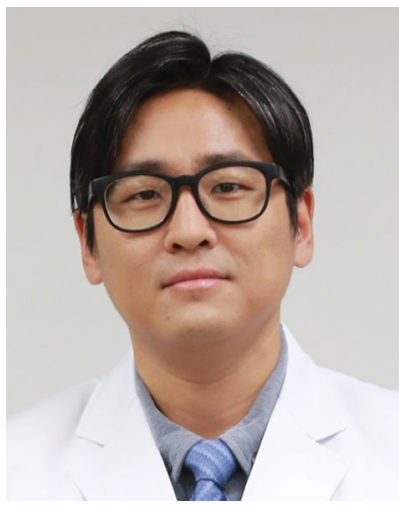
tween serious bleeding and atherothrombotic complications in these patients [1]. Recently, several randomized multicenter trials have demonstrated that compared with vitamin $\mathrm{K}$ antagonist (VKA)-based triple therapy (VKA in combination with a $\mathrm{P} 2 \mathrm{Y}_{12}$ inhibitor and acetylsalicylic acid [ASA]), dual therapy with non-vitamin $\mathrm{K}$ antagonist oral anticoagulant (NOAC) and a $\mathrm{P}_{2} \mathrm{Y}_{12}$ inhibitor was associated with a lower incidence of bleeding complications without increasing ischemic risks [2-5]. These trials focused on the first 6-14 months post-PCI and therefore do not inform physicians on the optimal strategy in AF patients with chronic coronary syndromes (CCS), defined as stable state beyond 1 year after PCI. Despite a lack of data regarding the benefits of oral anticoagulant (OAC: either VKA or NOAC) alone, the current guidelines recommend $\mathrm{OAC}$ monotherapy in $\mathrm{AF}$ patients with underlying $\mathrm{CCS}$ and previous PCI [1]. Dual therapy with an OAC and sin-

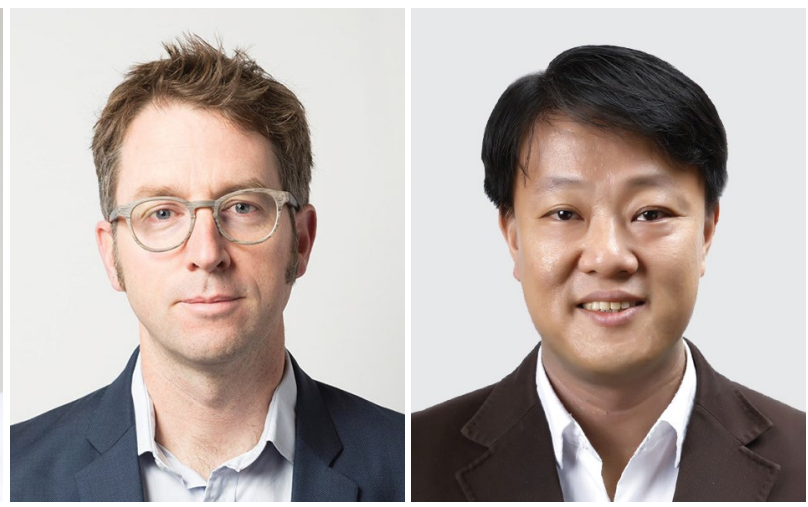

gle antipla-telet therapy (SAPT) (ASA or clopidogrel) may be considered only in selected cases with Complex High-Risk Indicated Procedure/ /Patients (CHIP) features such as complex PCI, diabetes, chronic kidney disease, or prior myocardial infarction (MI; class IIb).

Recently, two randomized clinical trials evaluating the optimal antithrombotic strategy in AF patients with CCS have been published [6, 7]. Optimizing Antithrombotic Care in Patients with Atrial Fibrillation and Coronary Stenting (OACALONE) failed to demonstrate non-inferiority of OAC monotherapy to a combined regimen with $\mathrm{OAC}$ and SAPT in AF patients with CCS beyond 1 year after coronary stenting, since this trial was underpowered and inconclusive as patient enrollment was prematurely terminated (following recruitment of only $36 \%$ of the prespecified sample size) [6]. Another randomized clinical trial, Atrial Fibrillation and Ischemic Events with

Address for correspondence: Young-Hoon Jeong, MD, PhD, Cardiovascular Center, Gyeongsang National University Changwon Hospital, 11 Samjeongja-ro, Seongsan-gu, Changwon 51472, Republic of Korea, e-mail: goodoctor@naver.com 
Table 1. Baseline characteristics and clinical outcomes in the OAC-ALONE and AFIRE trials [7, 8].

\begin{tabular}{|c|c|c|}
\hline & OAC-ALONE & AFIRE \\
\hline $\begin{array}{l}\text { OAC monotherapy vs. combined } \\
\text { OAC and SAPT }\end{array}$ & $\begin{array}{l}\text { OAC monotherapy }(n=344) \\
\text { vs. combined OAC and } \\
\text { SAPT }(n=346)\end{array}$ & $\begin{array}{l}\text { Rivaroxaban monotherapy } \\
(n=1107) \text { vs. combined } \\
\text { rivaroxaban and SAPT }(n=1108)\end{array}$ \\
\hline Study population & Japan & Japan \\
\hline Follow-up duration & 30 months & 23 months \\
\hline Age [year] & 74.9 vs. 75.2 & 74.3 vs. 74.4 \\
\hline Coronary stenting & $100 \%$ vs. $100 \%$ & $65.3 \%$ vs. $65.1 \%$ \\
\hline Left main coronary stenting & $6.7 \%$ vs. $6.4 \%$ & Not available \\
\hline Multivessel stenting & $34.6 \%$ vs. $35.0 \%$ & Not available \\
\hline $\mathrm{CHA}_{2} \mathrm{DS}_{2}$-VASc score & 4.6 vs. 4.6 & 4.0 vs. 4.0 (median) \\
\hline HAS-BLED score $\geq 3$ & $43.6 \%$ vs. $44.8 \%$ & $25.6 \%$ vs. $26.2 \%$ \\
\hline Combination regimen with ASA & $85.9 \%$ & $70.2 \%$ \\
\hline TTR (INR 2.0-3.0) for VKA & $54.9 \%$ vs. $47.9 \%$ & Not available \\
\hline $\begin{array}{l}\text { Ischemic endpoints } \\
\text { (for noninferiority) }\end{array}$ & $\begin{array}{c}15.7 \% \text { vs. } 13.6 \% \\
\text { (HR: } 1.16,95 \% \mathrm{Cl}: 0.79-1.72) \ddagger\end{array}$ & $\begin{array}{c}8.0 \% \text { vs. } 10.9 \% \\
\text { (HR: } 0.72,95 \% \mathrm{Cl}: 0.55-0.95) \S\end{array}$ \\
\hline $\begin{array}{l}\text { Bleeding endpoints } \\
\text { (ISTH major bleeding) }\end{array}$ & $\begin{array}{c}7.8 \% \text { vs. } 10.4 \% \\
\text { (HR: } 0.73,95 \% \mathrm{Cl}: 0.44-1.20)\end{array}$ & $\begin{array}{c}3.2 \% \text { vs. } 5.2 \% \\
\text { (HR: } 0.59,95 \% \mathrm{Cl}: 0.39-0.89 \text { ) superiority }\end{array}$ \\
\hline Myocardial infarction & $\begin{array}{c}2.3 \% \text { vs. } 1.2 \% \\
\text { (HR: } 2.03,95 \% \mathrm{Cl}: 0.64-7.59)\end{array}$ & $\begin{array}{c}1.2 \% \text { vs. } 0.7 \% \\
\text { (HR: } 1.60,95 \% \mathrm{Cl}: 0.67-3.87 \text { ) }\end{array}$ \\
\hline
\end{tabular}

${ }^{\ddagger}$ All-cause death, myocardial infarction, stroke, or systemic embolism

${ }^{5}$ All-cause death, myocardial infarction, stroke, systemic embolism, or unstable angina requiring revascularization

AFIRE - Atrial Fibrillation and Ischemic Events with Rivaroxaban in Patients with Stable Coronary Artery Disease; ASA - acetylsalicylic acid;

$\mathrm{Cl}$ - confidential interval; HR - hazard ratio; INR - international normalized ratio; ISTH - International Society on Thrombosis and Hemostasis; OAC — oral anticoagulant; OAC-ALONE - Optimizing Antithrombotic Care in Patients with Atrial Fibrillation and Coronary Stenting; SAPT — single antiplatelet therapy; TTR — time in therapeutic range; VKA — vitamin K antagonist

Rivaroxaban in Patients with Stable Coronary Artery Disease (AFIRE), evaluated the benefit of rivaroxaban monotherapy in $\mathrm{AF}$ patients with CCS who had undergone PCI or coronary artery bypass grafting more than 1 year earlier or who had a history of angiographically confirmed coronary artery disease without revascularization [7]. This trial demonstrated that compared with rivaroxaban plus SAPT combination therapy, the rivaroxaban monotherapy was non-inferior for prevention of ischemic events. In addition, rivaroxaban monotherapy showed a significantly lower rate of major bleeding and extrapolated to clinical practice. However, the results of those trials should be carefully interpreted in some points (Table 1). Firstly, the OAC-ALONE and AFIRE trials were conducted only in Japan. It is acknowledged that East Asian patients treated with anti-thrombotics are more vulnerable to bleeding complications when compared with Caucasians ("East Asian Paradox") [8]. In addition, the occurrence of MI related with AF appeared low in East Asian vs. Caucasian patients. Secondly, patients enrolled in the AFIRE trial received low dose rivaroxaban, approved in
Japan based on pharmacokinetic and clinical trials (15 $\mathrm{mg}$ or $10 \mathrm{mg}$ once daily) and warfarin was mainly administered in the OAC-ALONE trial, $(74.1 \%)$. In these studies, the preferred SAPT was ASA (86.4\% in OAC-ALONE and $70.2 \%$ in AFIRE) whereas clopidogrel is preferred in nonJapanese clinical practice. Therefore, a randomized multi-national trial will be required for patients with AF and CCS in Europe or America. Thirdly, not all patients in the AFIRE study had undergone previous PCI (only two-thirds) and procedural details were not described. Finally, it is important to acknowledge that the incidence of MI during follow-up was numerically higher in the OAC monotherapy groups in both trials $(0.93$ vs. 0.46 percent/patient-year in OAC-ALONE and 0.59 vs. 0.37 percent/patient-year in AFIRE, respectively).

In this issue of "Cardiology Journal", Franchina et al. [9] report a case review of ST-segmentelevation myocardial infarction (STEMI) in AF patients with CCS beyond 1 year after PCI, in the context of various antithrombotic regimens (OAC monotherapy in 3 cases, discontinuation of OAC due to a planned prostate biopsy in 1 case, 
combined OAC and SAPT in 1 case, and no antithrombotic therapy in 1 case). This case review reflects the "real-world" situation and challenges of antithrombotic strategy in daily practice. Despite OAC therapy, STEMI was observed in 4 patients, highlighting the complex and co-morbid nature of this patient population. High risk features included low left ventricular ejection fraction and mechanical valve replacement, and the majority of patients had multiple co-morbidities (hypertension [6 patients], diabetes mellitus [5 patients], dyslipidemia [4 patients], or smoking [current smoker in 3 patients and ex-smoker in 3 patients], respectively).

These anecdotal events and the numerical increase in MI observed with OAC monotherapy in the most contemporary trials provide persisting uncertainty regarding the optimal antithrombotic strategy for AF patients with $\mathrm{CCS}$ and previous PCI. Prolongation of combined OAC and SAPT may provide protection against MI but a delicate balance between ischemic and bleeding complications remains. Ideally, a tailored antithrombotic strategy should be determined according to a balanced evaluation of PCI complexity (e.g., CHIP scoring), bleeding risk (e.g. High Bleeding Risk criteria [10]) and a patients' hemostatic measurement (e.g., coagulation activity, platelet activation), which would be the right road to "precision medicine".

Funding: This study is supported by research grants from AstraZeneca Korea and the Basic Science Research Program through the National Research Foundation (NRF) of Korea funded by the Ministry of Science, ICT and Future Planning (NRF-2015R1A5A2008833).

Conflict of interest: Young-Hoon Jeong reports honoraria for lectures from AstraZeneca, SanofiAventis, Daiichi Sankyo, Otsuka, Han-mi Pharmaceuticals, and Yuhan Pharmaceuticals, and research grants or support from AstraZeneca, Han-mi Pharmaceuticals, Yuhan Pharmaceuticals, and Haemonetics; Thomas W. Johnson reports honoraria for lectures/consultancy from AstraZeneca and Bayer; Yongcheol Kim - no conflict of interest.

\section{References}

1. Lip GYH, Collet JP, Haude M, et al. 2018 Joint European consensus document on the management of antithrombotic therapy in atrial fibrillation patients presenting with acute coronary syndrome and/ /or undergoing percutaneous cardiovascular interventions: a joint consensus document of the European Heart Rhythm Association (EHRA), European Society of Cardiology Working Group on Thrombosis, European Association of Percutaneous Cardiovascular Interventions (EAPCI), and European Association of Acute Cardiac Care (ACCA) endorsed by the Heart Rhythm Society (HRS), Asia-Pacific Heart Rhythm Society (APHRS), Latin America Heart Rhythm Society (LAHRS), and Cardiac Arrhythmia Society of Southern Africa (CASSA). Europace. 2019; 21(2): 192-193, doi: 10.1093/europace/euy174, indexed in Pubmed: 30052888.

2. Gibson CM, Mehran R, Bode C, et al. Prevention of Bleeding in Patients with Atrial Fibrillation Undergoing PCI. N Engl J Med. 2016; 375(25): 2423-2434, doi: 10.1056/NEJMoa1611594, indexed in Pubmed: 27959713.

3. Cannon C, Bhatt D, Oldgren J, et al. Dual antithrombotic therapy with dabigatran after PCI in atrial fibrillation. N Engl J Med. 2017; 377(16): 1513-1524, doi: 10.1056/nejmoa1708454.

4. Lopes R, Heizer G, Aronson R, et al. Antithrombotic Therapy after Acute Coronary Syndrome or PCI in Atrial Fibrillation. N Engl J Med. 2019; 380(16): 1509-1524, doi: 10.1056/nejmoa1817083.

5. Vranckx P, Valgimigli M, Eckardt L, et al. Edoxaban-based versus vitamin $\mathrm{K}$ antagonist-based antithrombotic regimen after successful coronary stenting in patients with atrial fibrillation (ENTRUST-AF PCI): a randomised, open-label, phase $3 \mathrm{~b}$ trial. Lancet. 2019; 394(10206): 1335-1343, doi: 10.1016/S01406736(19)31872-0, indexed in Pubmed: 31492505.

6. Matsumura-Nakano Y, Shizuta S, Komasa A, et al. Open-Label Randomized Trial Comparing Oral Anticoagulation With and Without Single Antiplatelet Therapy in Patients With Atrial Fibrillation and Stable Coronary Artery Disease Beyond 1 Year After Coronary Stent Implantation. Circulation. 2019; 139(5): 604-616, doi: 10.1161/circulationaha.118.036768.

7. Yasuda S, Kaikita K, Akao M, et al. AFIRE Investigators. Antithrombotic therapy for atrial fibrillation with stable coronary disease. N Engl J Med. 2019; 381(12): 1103-1113, doi: 10.1056/ NEJMoa1904143, indexed in Pubmed: 31475793.

8. Huo Y, Jeong YH, Gong Y, et al. 2018 update of expert consensus statement on antiplatelet therapy in East Asian patients with ACS or undergoing PCI. Science Bulletin. 2019; 64(3): 166-179, doi: 10.1016/j.scib.2018.12.020.

9. Franchina AG, Calderone D, D'Arrigo P, et al. Mechanisms of STsegment elevation myocardial infarction in patients with atrial fibrillation, prior stenting and long-standing chronic coronary syndrome. Cardiol J. 2020; 27(1): 8-15, doi: 10.5603/CJ.a2020.0002, indexed in Pubmed: 31960945.

10. Urban P, Mehran R, Colleran R, et al. Defining high bleeding risk in patients undergoing percutaneous coronary intervention. Circulation. 2019; 140(3): 240-261, doi: 10.1161/CIRCULATIONAHA.119.040167, indexed in Pubmed: 31116032. 